\title{
Effect of a Splitter Plate on the Dynamics of a Vortex Pair
}

\author{
Jason Rabinovitch ${ }^{1}$ \\ Graduate Aerospace Laboratories, California Institute of Technology, Pasadena, California, 91125 \\ Vincent Brion ${ }^{2}$ \\ ONERA - Departement d'Aerodynamique Fondamentale et Experimentale, Meudon, 92190, France \\ and \\ Guillaume Blanquart ${ }^{3}$ \\ Mechanical Engineering, California Institute of Technology, Pasadena, California, 91125
}

An experimental and numerical study was performed to investigate the behavior of a counter-rotating vortex pair as well as a single vortex in the vicinity of a wall. A wind tunnel with two NACA0012 profiles mounted vertically with an optional splitter plate in the center and a 3D PIV system were used to experimentally study the interactions between two counter-rotating vortices, as well as the interactions between a vortex and a wall. Many fundamental differences were found between the two configurations, which promote the growth of the Crow instability in the two vortex configuration, but not in the one vortex/wall configuration. The numerical results obtained re-enforced the experimental results, and emphasize the fundamental physical differences between the two configurations. While modeling a vortex wall system with an image vortex may give correct integral results for loads experienced by blades, this model does not accurately describe the downstream dynamics of the vortex system.

\section{Nomenclature}

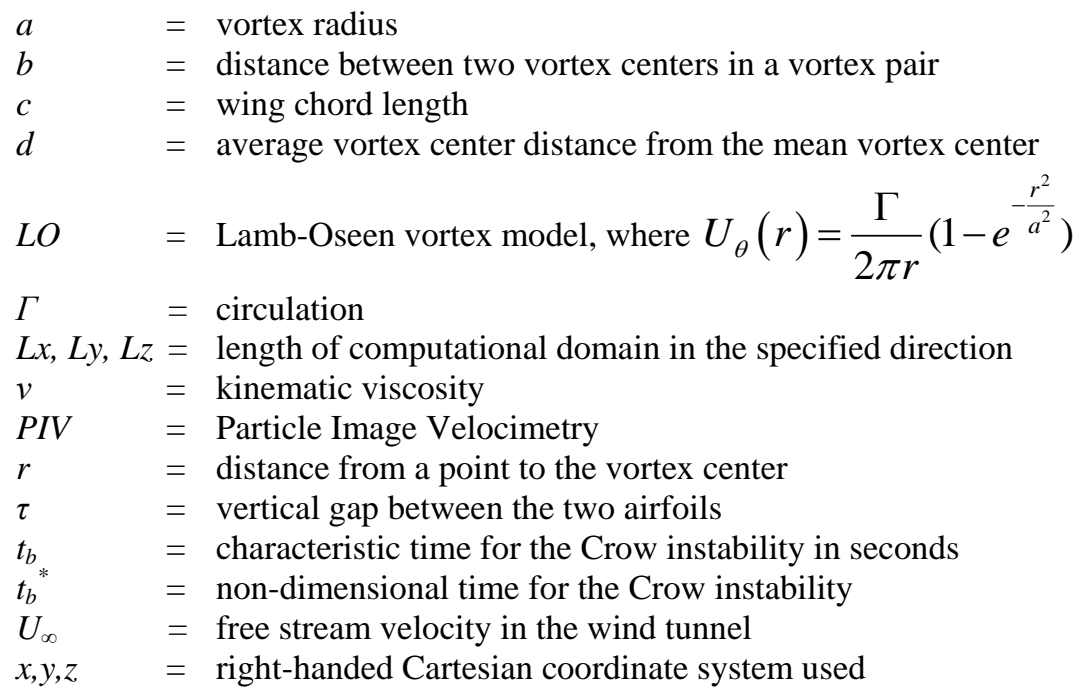

\footnotetext{
${ }^{1}$ Graduate Student, AIAA Student Member.

${ }^{2}$ Research Engineer, AIAA Member.

${ }^{3}$ Assistant Professor of Mechanical Engineering, AIAA Member.
} 


\section{Introduction}

$\mathrm{C}$ ounter-rotating vortex pairs have received a lot of attention in the aerodynamic community. The most common occurrence of these vortices are "wing-tip vortices," which dominate the behavior of the wake of an airplane. To avoid endangering following aircrafts, a minimum separation time is enforced between take-offs and landings based on the relative size of the two aircrafts. If the time needed for the wing-tip vortices to decay could be shortened, airport capacity could be greatly increased, while still maintaining the same margin of safety. One of the most well known interactions between these vortices is the Crow instability, which is a long wave vortex instability. ${ }^{1}$ While other vortex instabilities exist for a counter-rotating vortex pair, the Crow instability is generally considered to be the primary mechanism for the break-up and dissipation of the vortices. ${ }^{2}$

In turbo machinery, the relative motion of the rotor blades and the stationary wall produces a vortex, often referred to as a tip leakage vortex. The resulting system is similar to the wing-tip vortices produced behind an airplane. Schematics of this process are shown in Fig. 1.1. Similar to wing tip vortices, the pressure difference between the suction side and pressure side of the blade induces a flow that eventually rolls up into a single vortex. The characteristics of the vortex are heavily influenced by the aerodynamics of the blade as well as by the gap between the blade and the wall. Previous studies have shown that the mechanism for the

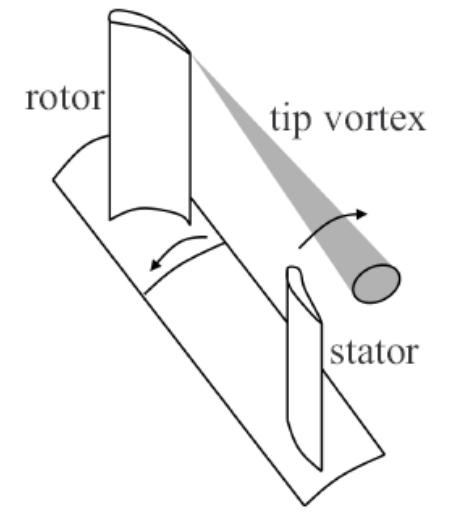

Figure 1.1. Schematic photos of a tip leakage vortex. ${ }^{3}$

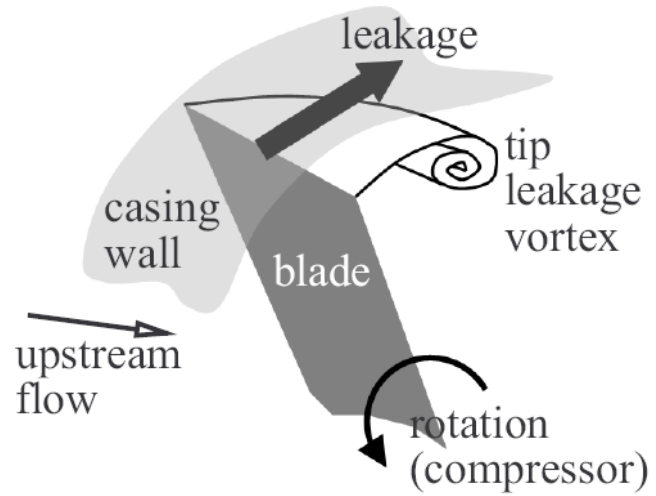

(compressor)

formation of a tip leakage vortex is primarily inviscid. ${ }^{4}$ Under these conditions, an image vortex might be used to replace the wall, resulting in a situation similar to trailing wing-tip vortices. ${ }^{5}$ Understanding the tip leakage flow as well as the behavior of the flow downstream of the rotor could lead to more efficient compressor designs.

The purpose of this study is to investigate the Crow instability in two flow configurations, namely with a split wing apparatus (two-vortex configuration) and a single wing with a splitter plate (one-vortex configuration). In both cases, the Crow instability could be used to encourage vortex break-down and increase the efficiency of the systems. Two different instabilities occur as a result of the interaction between two counter-rotating vortices: a shortwavelength instability and a long-wavelength instability. The short wavelength fluctuation is referred to as the Widnall instability ${ }^{6}$ the long wave length instability is referred to as the Crow instability. ${ }^{1}$ The Crow instability is considered to be the most important instability contributing to the eventual decay of the vortices, and will be the primary focus of this work.

A distinct characteristic frequency is associated with the Crow instability $(\sim 150 \mathrm{~Hz}$ for the current experimental set-up). Unfortunately, this frequency is too high to be captured by the PIV system used (4 Hz). On the other hand, due to the nature of the instability, the vortex centers have a theoretical preferred direction of oscillation which is along a $45^{0}$ angle with the plane of symmetry. With accurate vortex center detection methods, it may be possible to detect the presence/absence of the Crow instability by determining whether or not the vortex centers are aligned along a $45^{\circ}$ line with the flow. Time scales associated with this instability are presented in Appendix A.

The overall objective of this study is to compare and contrast the dynamics of a counter-rotating vortex pair and a tip-leakage vortex, specifically focusing on the Crow instability. This analysis will be performed both experimentally and numerically. In the following sections, the experimental apparatus will be described, followed by the post-processing techniques used to analyze the data taken. An overview of the results obtained (both experimental and numerical) will be presented, along with a discussion of these results. Finally, the article summarizes currently ongoing work and highlights the need for future work.

\section{Experimental Methodology}

The two-vortex and one-vortex configurations were investigated using PIV in a wind tunnel. This section briefly explains the experimental equipment used as well as the vortex center detection methods. 


\section{A. Wind tunnel and Experimental Apparatus}

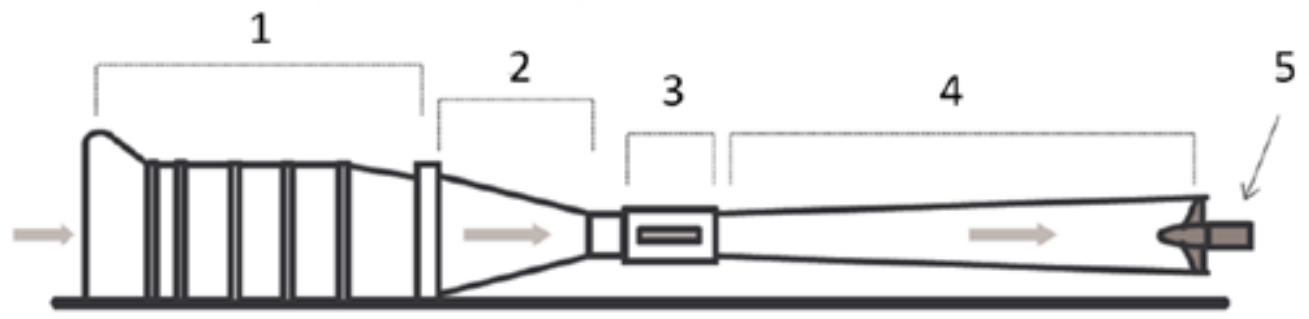

Figure 2.1. Schematic drawing of the wind tunnel used. 1 - Settling chamber 2 Converging section 3 - Test section 4 - Diffuser 5 - Axial Fan. Note that the flow is going from left to right.

Figure 2.1 shows a schematic diagram of the wind tunnel used for all experiments. It is an open circuit wooden wind tunnel, with a circular test section. The possible speeds that can be attained at the entrance to the test section range from approximately $20 \mathrm{~m} / \mathrm{s}$ to $43 \mathrm{~m} / \mathrm{s}$.

Figure 2.2 shows a basic schematic of the experimental set-up found in the test section. The experimental set-up consists of two NACA0012 wings mounted vertically in the middle of the test section. Each wing can be rotated, and the angle of attack can vary between $-15^{\circ}$ and $+15^{\circ}$. In addition, the wings can be moved vertically, so that the gap $(\tau)$ between the wings can be varied from 0 to $50 \mathrm{~mm}$.

Two configurations are possible with this set-up as a splitter plate can be added or removed from the test section. Configuration 1 (C1) will refer to experiments where the splitter plate is present. In the $\mathrm{C} 1$ configuration, $\tau$ refers to twice the gap between the airfoil and the splitter plate, in order to remain consistent with the gap size defined for the second configuration (C2). This first configuration is used to study the interaction between a single vortex and a wall. Configuration 2

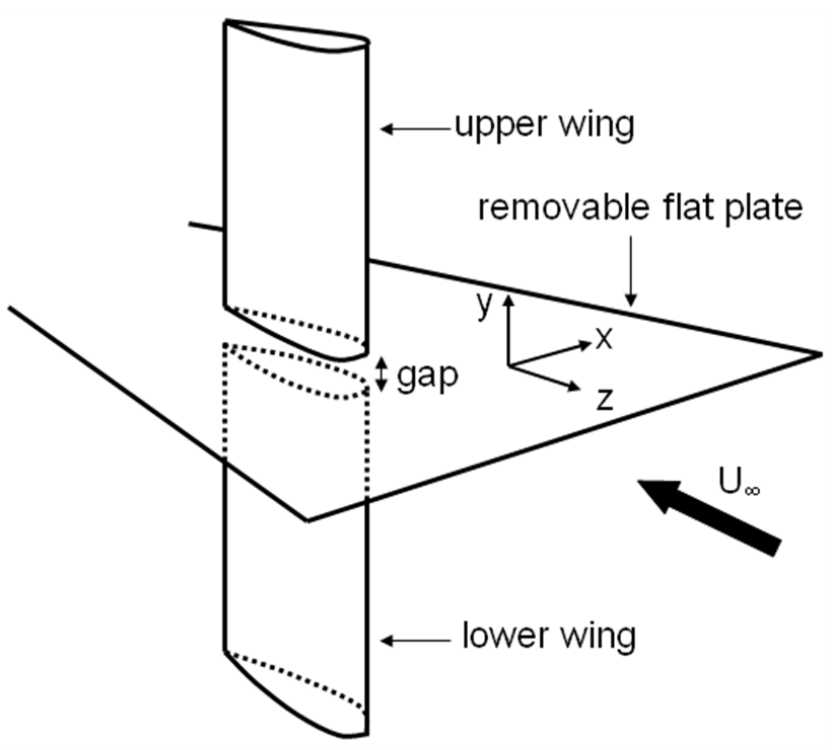

Figure 2.2. Basic schematic of the experimental set-up.

(C2) will refer to experiments where the splitter plate is not present. This configuration is used to study vortex pair interactions, as two wing-tip vortices are produced and interact with each other. The results from the $\mathrm{C} 1$ configuration are compared and contrasted to the results obtained from the $\mathrm{C} 2$ configuration. Figure 2.3 shows pictures taken of the two different experimental setups. 


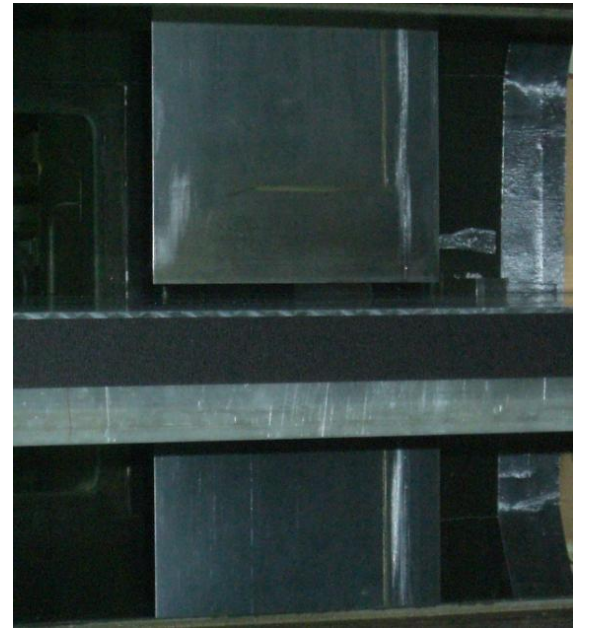

a)

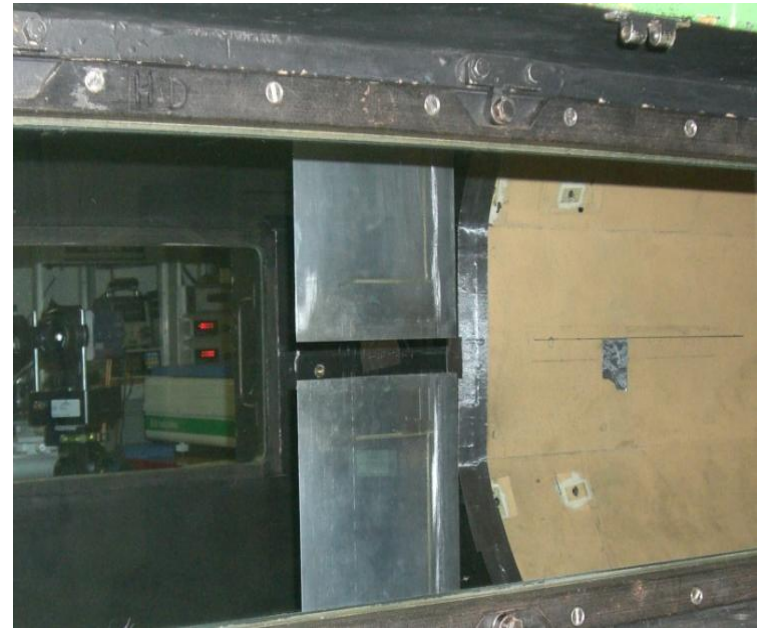

b)

Figure 2.3. Photos of the test section. a) C1. b) C2.

The free stream velocity in the test section $\left(U_{\infty}\right)$ was $39.5 \pm 0.5 \mathrm{~m} / \mathrm{s}$. All distances were normalized by the chord length, $c$, and times were normalized by $c / U_{\infty}$. A Reynolds number based on the chord length $\left(\operatorname{Re}_{c}\right)$ is defined as

$$
\operatorname{Re}_{c}=\frac{U_{\infty} c}{v}
$$

This corresponds to a Reynolds number of approximately 550,000. A summary of important parameters corresponding to the apparatus is given in Table 2.1.

\begin{tabular}{|c|c|}
\hline \multicolumn{2}{|c|}{ Important Characteristics } \\
\hline Foil Shape & NACA0012 \\
Chord, $c$ & $0.2 \mathrm{~m}$ \\
Span (Approx) & $0.4 \mathrm{~m}$ \\
Angle & $-15^{\circ}$ to $+15^{\circ}$ \\
Gap, $\tau$ & 0 to $50 \mathrm{~mm}$ \\
\hline
\end{tabular}

Table 2.1

To ensure that all boundary layers are fully turbulent, Carborundum grains were used based on the model given by Schlichting. ${ }^{7}$ Type 80 Carborundum grains were placed at $0.25 c$ along the splitter plate, as well as at $0.17 c$ along the airfoils.

Figure 2.4 shows the general schematic for the PIV system used in this experiment, and a summary of important PIV characteristics is given in Appendix B. For this investigation, measurements were taken at 4 different downstream locations: $2.0 c, 2.5 c, 3.0$ $c$, and $4.0 c(40 \mathrm{~cm}, 50 \mathrm{~cm}, 60 \mathrm{~cm}$, and $80 \mathrm{~cm}$, respectively). The distance downstream is measured in the direction of the flow, from the trailing edge of the airfoils when at $0^{0}$ angle of attack. A

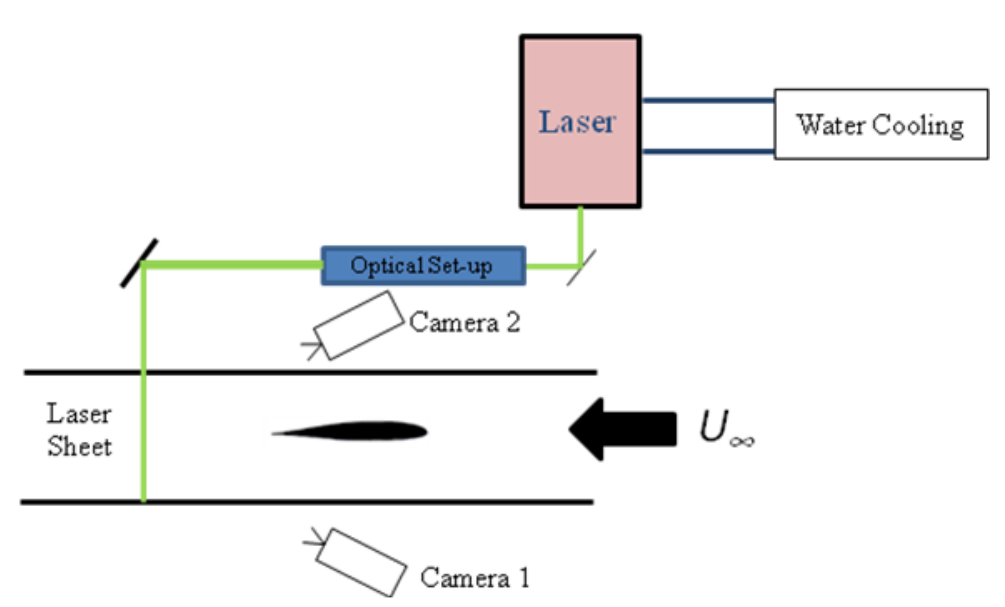

Figure 2.4. Schematic of test section for PIV as seen from above. Note that the smoke generator is placed next to the axial fan, at the exit of the wind tunnel, and not at the intake. 
range of gap sizes and angles of attack were considered. The gap size, $\tau$, ranged from $18 \mathrm{~mm}$ to $50 \mathrm{~mm}$, and the angle of attack ranged from $4^{0}$ to $12^{0}$. In the interest of brevity, all of the data presented in Section III had a constant gap size of $30 \mathrm{~mm}$, and an angle of attack of $8^{0}$. These data were then taken at different downstream locations.

\section{B. Vortex Center Detection Methods}

Due to the relatively low frequency of the PIV system used $(4 \mathrm{~Hz})$ and the high predicted frequency of the Crow instability $(150 \mathrm{~Hz})$, the most accurate way to detect the presence of the Crow instability is to use the center locations of the instantaneous snapshots taken. A $45^{\circ}$ oscillation of the vortex centers is expected if the instability is present. Such analysis requires a reliable and robust method to detect the vortex center from an instantaneous PIV vector field, with limited signal-tonoise ratio.

Many different potential center detection algorithms were investigated in order to determine the most appropriate method for this study. A robust, efficient, and easily automated algorithm was desired as this method would be applied to large numbers of datasets of instantaneous PIV snapshots (1000 or 2000 images at a

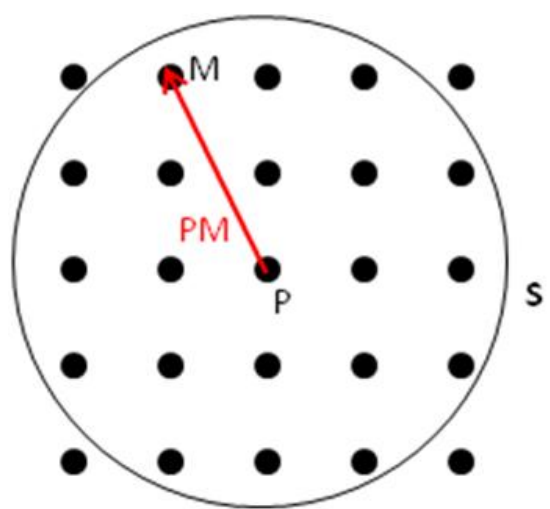

Figure 2.5. Representation of the different vectors needed for the $\Gamma_{1}$ and $\Gamma_{2}$ methods.

time). In an effort to reduce the influence of erroneous vectors, methods requiring no interpolation or calculations of derivatives were preferred. Both the $\Gamma_{1}$ and $\Gamma_{2}$ methods $^{8}$ seemed to be the most promising of pre-existing methods, especially when compared to the $\mathrm{Q}$ criterion ${ }^{9}$, and maximum vorticity methods ${ }^{9}$.

For the maximum vorticity method, the vorticity field was calculated for every snapshot, and the center was taken to be the point with the largest magnitude of vorticity. With this method, one erroneous vector could create a large spike in vorticity, which would cause an incorrect center to be detected. The Q method involves many gradients to be calculated, and due to noise in the PIV velocity fields, the results were not found to be reliable for this study.

Graftineaux defined two quantities that can be used to calculate vortex centers and to identify points inside of a vortex, known as $\Gamma_{1}$ Eq. (2.2) and $\Gamma_{2}$ Eq. (2.3)

$$
\Gamma_{1}(P)=\frac{1}{N} \sum_{S} \frac{\left(P M \wedge U_{M}\right) \cdot z}{\|P M\| \cdot\left\|U_{M}\right\|},
$$

where $P$ is a fixed point in the measurement domain. $S$ is a two-dimensional area surrounding $P$, referred to as the window size, whose size must be defined by the user. $N$ is the number of data points $(M)$ that are located within $S$, and $U_{M}$ is the velocity vector at point $M$. Finally, $z$ is a unit vector normal to the plane of measurement, and. $\Gamma_{1}$ (or $\Gamma_{2}$ ) is calculated for all points in the measurement domain and then used to determine the center of the vortex. For an ideal axisymmetric vortex, the maximum value of $\left|\Gamma_{1}\right|$ is 1 . For each possible center position, the $\Gamma_{1}$ criterion calculates the degree to which the flow rotates around this point. In this work, near the vortex core, $\left|\Gamma_{1}\right|$ was found to reach values between 0.9 and 1. A diagram for this method is shown in Fig. 2.5.

The $\Gamma_{2}$ method is a variant of the $\Gamma_{1}$ method and the criterion for $\Gamma_{2}$ is given by Eq. (2.3).

$$
\Gamma_{2}(P)=\frac{1}{N} \sum_{S} \frac{\left(P M \wedge\left(U_{M}-\bar{U}_{P}\right) \cdot z\right.}{\|P M\| \cdot\left\|U_{M}-\bar{U}_{P}\right\|}
$$

The only difference between the $\Gamma_{1}$ and $\Gamma_{2}$ criteria is that the average velocity over the window, $\overline{U_{p}}$ is subtracted away. This takes into account any uniform flow within the plane of rotation. With this method, when $\left|\Gamma_{2}\right|$ is greater than approximately $2 / \pi, P$ is assumed to represent a point in the vortex.

In this experiment, the $\Gamma_{2}$ method was used initially instead of the $\Gamma_{1}$ method, and the center of the vortex was identified to be the point where $\left|\Gamma_{2}\right|$ was the largest. As long as the window size chosen was on the order of the 
calculated vortex radius, or smaller, the window size did not play a large role in determining the vortex center. This method also has the advantage that $\Gamma_{2}$ will change signs for different signs of vorticity, and no gradients need to be calculated.

Based on the $\Gamma_{2}$ method, a new center detection algorithm was developed. For each snapshot taken, the circulation was calculated at all points in the domain by using a square integration window around each point. The center was taken to be the point in the domain which had the largest circulation. A square integration window was used to avoid additional interpolations when calculating the circulation, $\Gamma=\oint u \cdot d l$. Circulation was calculated using a line integral instead of an area integral with vorticity in order to avoid calculating derivatives of the velocity field. Interestingly, the integration was found to smooth out some of the inherent noise in the velocity field. This method gave extremely similar results to the $\Gamma_{2}$ methods, but was computationally cheaper to perform.

In order to test this method, a velocity field corresponding to an ideal Lamb-Oseen vortex was generated for the same mesh spacing used in the experiment. White Gaussian noise was added to this ideal profile, and the above described circulation center detection method was applied. Average values for circulation and radius were used to simulate the experimental conditions as best as possible. It was found that when the average magnitude of the white noise was $10 \%$ of the maximum vortex velocity, the average error in the center detection in $\mathrm{x}$ and $\mathrm{y}$ was 0.1 grid points.

\section{Experimental Results and Discussion}

In this section, the experimental results are presented, showing the effect of vortex meandering, sample velocity fields, center distributions, and time scales associated with the Crow instability. In addition, differences between the two configurations are discussed.

\section{A. Vortex Meandering}

In order to accurately describe the physics of a system when averaging PIV instantaneous snapshots, the phenomenon of vortex meandering must be taken into account. Vortex meandering refers to the seemingly random and slow oscillation of a vortex center for a vortex that has been produced in a wind tunnel. There is no preferred direction of oscillation nor characteristic frequency associated with this phenomenon. For any time averaged quantities calculated from PIV images, vortex meandering will cause the results to inaccurately describe the characteristics of the true vortex being studied. For example, the movement of the vortex centers will cause the vortex radius calculated from an averaged image to be larger than it should be, as the meandering effect effectively obscures the true nature of the vortex. Many attempts have been made to quantify the effect of this meandering on the vortex. Devenport has done much work on this subject, and after careful modeling, it was found that due to meandering the core radius is around 10\% smaller than indicated, the tangential velocity should be around $15 \%$ higher, and the velocity deficit in the axial direction should be around $10 \%$ higher (this was calculated at a distance $30 c$ downstream). ${ }^{10}$ Although the Crow instability has a preferred direction of oscillation for the vortex centers, the overall effect of this instability on calculated averaged PIV quantities will be similar to the effect of vortex meandering.

In order to take this effect into account, all of the instantaneous snapshots were shifted so that each image was centered on the same grid point. Then, the velocity field was averaged in an attempt to analyze the true characteristics of the vortex. Figure 3.1 shows how the oscillating center of the vortex affects the values calculated from an averaged velocity field. Here, a horizontal cut is taken along the centerline of the vortex from the averaged PIV field. The y-coordinate is held constant in the plane and taken at the center of the vortex, and the vertical component of velocity is plotted. For the data represented by the dotted line, no corrections were made to the averaged velocity field calculated. For the solid line data, each individual snapshot was shifted so that all calculated centers lined up with each other before the average velocity field was calculated. The effect of both the Crow instability (which was present in this trial) and vortex meandering can clearly be seen. By shifting all vortex centers, the vortex radius has been decreased, and the maximum azimuthal velocity has been increased, consistent with predictions from Devenport. ${ }^{10}$ Farther downstream, re-centering all of the images has a larger effect, as the oscillations from both vortex meandering and the Crow instability increase in magnitude as one travels downstream. When this algorithm was applied to circulation calculations, the circulation of a vortex was observed not to change if the circulation was calculated far enough away from the vortex center. 


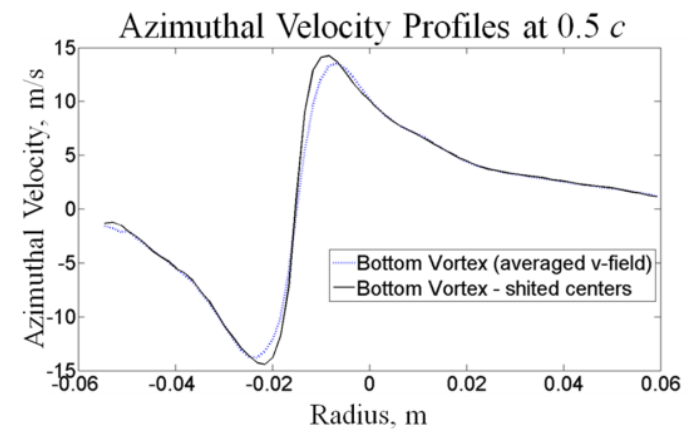

a)

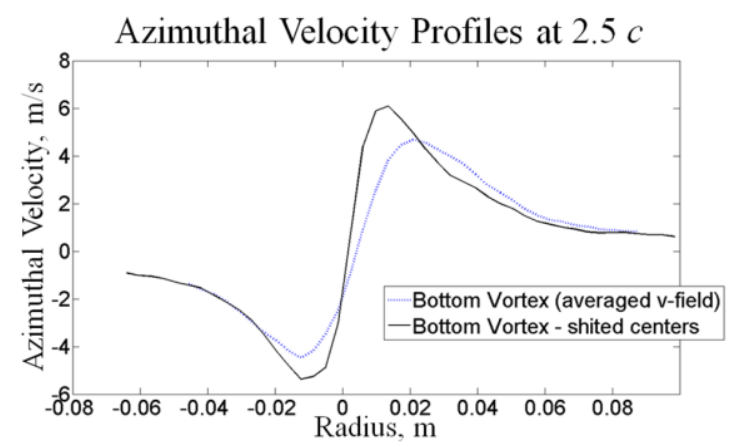

b)

Figure 3.1. Results comparing shifted and non-shifted centers for $0.5 c$ downstream (a) and $2.5 c$ downstream (b).

\section{B. Vortex Dynamics}

Figure 3.2 shows two sample averaged in-plane vector fields measured experimentally. Figure 3.2 a) shows two counter-rotating vortices, while Fig. 3.2 b) shows one vortex close to the splitter plate.

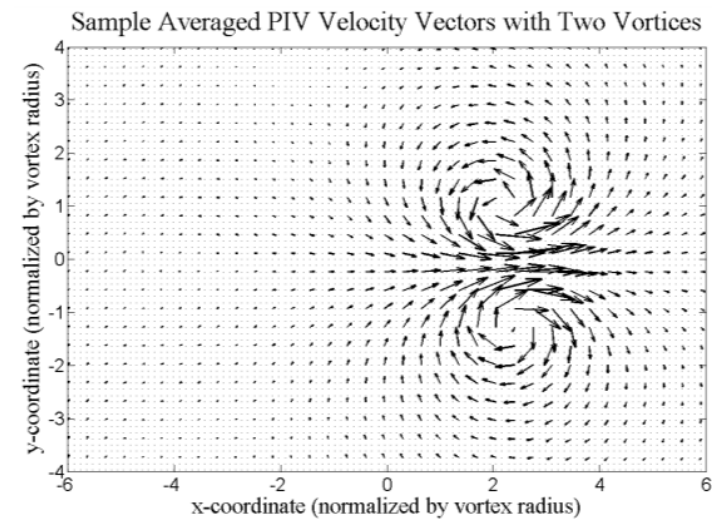

a)

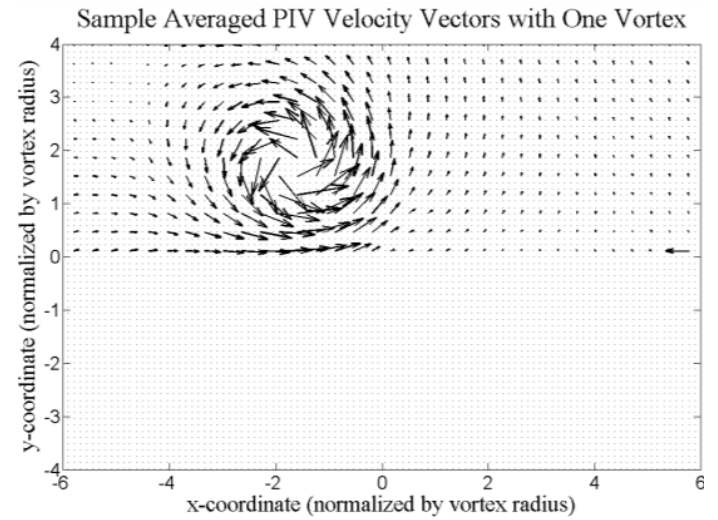

b)

Figure 3.2. Sample averaged in plane velocity vectors taken at $2.5 c$ downstream, $\tau=30 \mathrm{~mm}$, and a free stream velocity of $39.5 \mathrm{~m} / \mathrm{s}$. (a) shows C2 (2000 images averaged), and (b) shows C2 (1000 images averaged).

Figure 3.3 shows a contour plot representing the frequency at which the center of the vortex falls on a specific grid point for one data series. The colors refer to counts of how many times the same center positions were found during one experiment.

In Fig. $3.3 \mathrm{a}$ ), the two-vortex configuration is shown where the Crow instability is present, while in $3.3 \mathrm{~b}$ ), the one vortex configuration is shown where the Crow instability is not present. Once again, the presence of the Crow instability is determined by a preferred angle of oscillation of the vortex centers. 


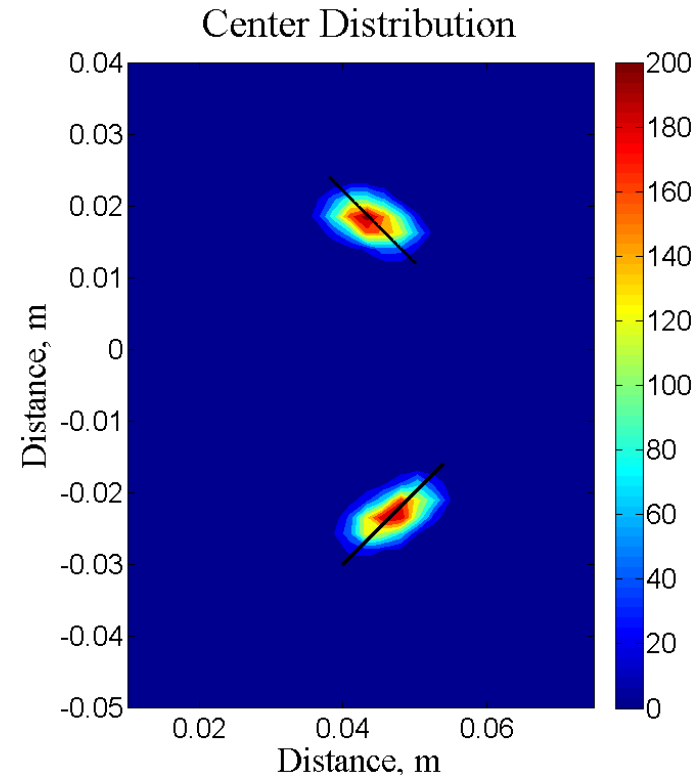

a)

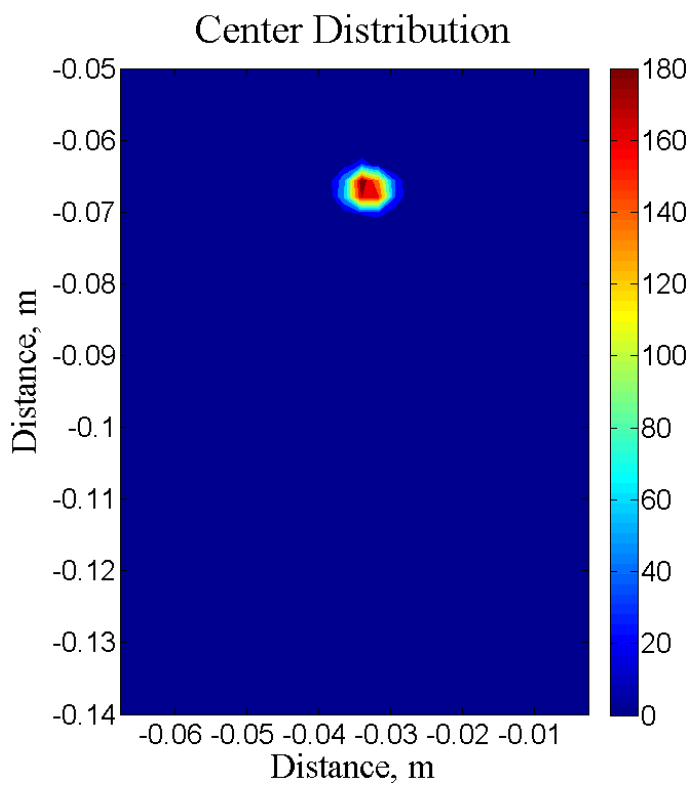

b)

Figure 3.3. Contour plots showing the distribution of centers for $\tau=30 \mathrm{~mm}, 2.5$ downstream and a free stream velocity of $39.5 \mathrm{~m} / \mathrm{s}$. (a) shows C2 (2000 images used), and (b) shows C1 (1000 images used). Black lines have been drawn at $45^{\circ}$ manually showing the predicted preferred direction of the center oscillation.

While it is possible to identify visually whether or not the Crow instability is present, a more quantitative analysis was used to estimate the preferred angle of oscillation. More precisely, a principal component analysis was used in order to determine the primary direction of oscillation of the vortex centers. ${ }^{11}$ These results are summarized in Table 3.1.

Principal Component Analysis Results

\begin{tabular}{|c|c|c|c|}
\hline Downstream Location & Vortex Location & $\begin{array}{c}\text { Angle (degrees, shifted } \\
\text { to lie between 0 } \\
\text { and } \\
\mathbf{9 0}\end{array}$ & $\begin{array}{c}\text { Normalized } \\
\text { Eigenvalue }\end{array}$ \\
\hline $2.0 \mathrm{c}$ & Top & 70 & 0.75 \\
\hline $2.0 \mathrm{c}$ & Bottom & 60 & 0.78 \\
\hline $2.5 \mathrm{c}$ & Top & 70 & 0.77 \\
\hline $2.5 \mathrm{c}$ & Bottom & 62 & 0.8 \\
\hline $3.0 \mathrm{c}$ & Top & 70 & 0.75 \\
\hline $3.0 \mathrm{c}$ & Bottom & 60 & 0.77 \\
\hline $4.0 \mathrm{c}$ & Top & 76 & 0.66 \\
\hline $4.0 \mathrm{c}$ & Bottom & 66 & 0.69 \\
\hline
\end{tabular}

Table 3.1

In Table 3.1, the angle is defined as the angle made between a vertical line (aligned with a vertical line in Fig. 3.3 a) and the primary direction of oscillation of the vortex center. The normalized eigenvector (maximum possible value of 1) is a measure of how much of the vortex movements is in this primary direction. Movement in other directions can be attributed to vortex meandering, the turbulent flow field, etc. Although the predicted theoretical value of $45^{\circ}$ is not observed, the two angles do remain fairly constant, at approximately $70^{\circ}$ and $60^{\circ}$. In addition, the relatively high eigenvalues indicate that the majority of movement of the vortex centers occurs along this direction, which we can attribute to the Crow instability.

\section{Differences between the two Configurations}

Many differences were observed between the $\mathrm{C} 1$ and $\mathrm{C} 2$ configurations, and these contributed to detecting the Crow instability only for the $\mathrm{C} 2$ configuration and not for the $\mathrm{C} 1$ configuration. 
When the Crow instability is present, the centers are expected to oscillate much more than when the Crow instability is not present. If the Crow instability is not present, the center oscillations can be attributed to vortex meandering effects. ${ }^{10}$ One way to estimate this is to calculate an average distance from the mean center position, using:

$$
d=\frac{1}{N} \sum_{i=1}^{N} \sqrt{\left(x_{i}-x_{\text {mean }}\right)^{2}+\left(y_{i}-y_{\text {mean }}\right)^{2}}
$$

where $x_{i}$ and $y_{i}$ refer to the centers found from the circulation method for each snapshot, and $N$ images have been taken at a particular downstream location. The evolution of this mean distance as a function of downstream location is shown in Fig. 3.4 for both configurations. As expected, this mean distance increases with downstream distance, and the overall growth observed is much larger when the Crow instability is present, further confirming that the Crow instability is not present in the $\mathrm{C} 1$ configuration.

One fundamental difference between the two configurations is the discrepancy in characteristic times for the Crow instability to develop $\left(t_{b}{ }^{*}\right)$, as illustrated in Fig. 3.5. This large difference in $t_{b}{ }^{*}$ explains why the

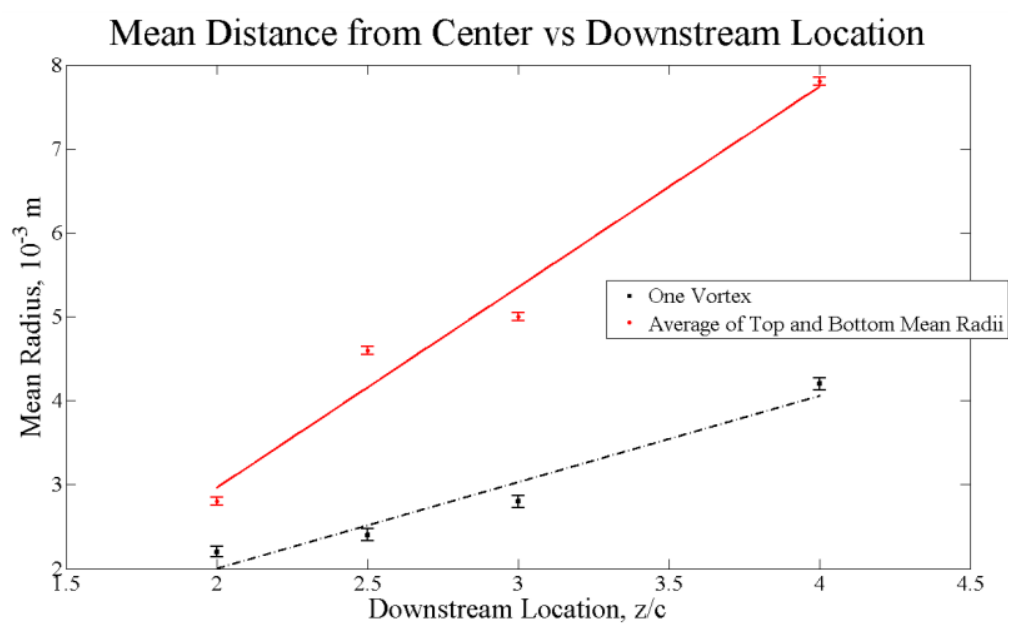

Figure 3.4. Average distance of the instantaneous vortex centers to the mean center. When two vortices are present, the radius for the top and bottom vortices are averaged.

dynamics of the two systems are so different. In an attempt to promote the Crow instability in the C1 configuration, the experimental parameters were varied in order to try to reduce $t_{b}{ }^{*}$ and match the $t_{b}{ }^{*}$ for the two vortex case shown in Fig. 3.3.

Unfortunately, matching the characteristic times between the two configurations proved to be much more challenging than expected. With the experimental apparatus, it was possible to change both the angle of attack as well as the gap size. However, increasing the gap size increased $b$ but also increased $\Gamma$. The opposite was seen if the gap size was decreased. The angle of attack did not have a large effect on the characteristic time. This led to a characteristic time that was fairly constant for the $\mathrm{C} 1$ configuration, and not low enough for the Crow instability to have time to develop.

Another physical difference between the two configurations is the effect of viscosity along the splitter plate. Due to the no-slip condition, a boundary layer along the splitter plate in the cross-stream direction is created as well as a boundary layer in the streamwise direction. This in turn creates a region of opposite signed vorticity near the wall, which is convected away from the wall by the vortex. This effect is shown in Fig. 3.6. These two regions of opposite signed vorticity cause a rebound effect, increasing the distance between the vortex and the wall (increase in $b$ ).

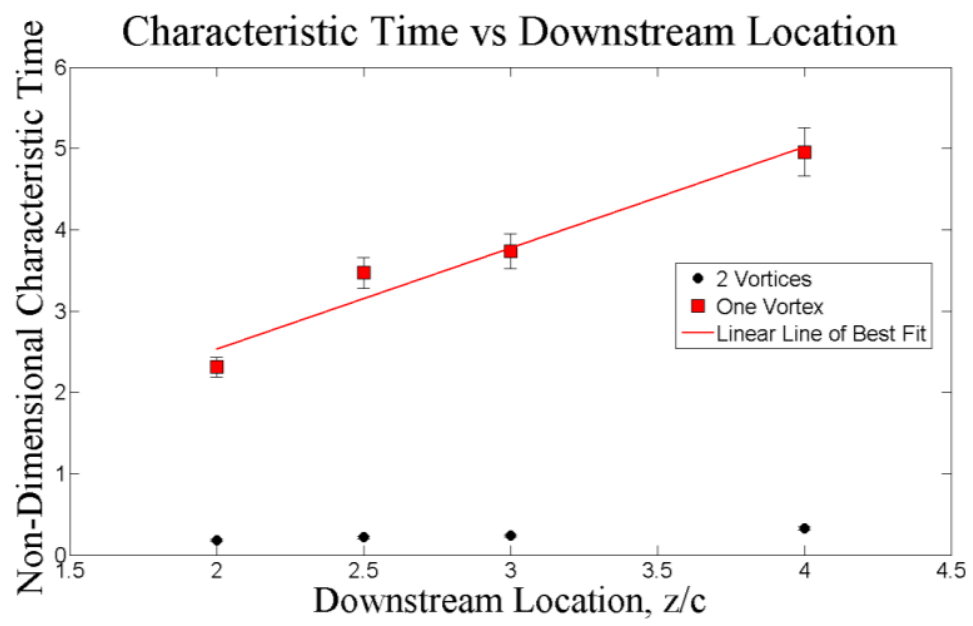

Figure 3.5. Evolution of the characteristic time vs downstream location. 


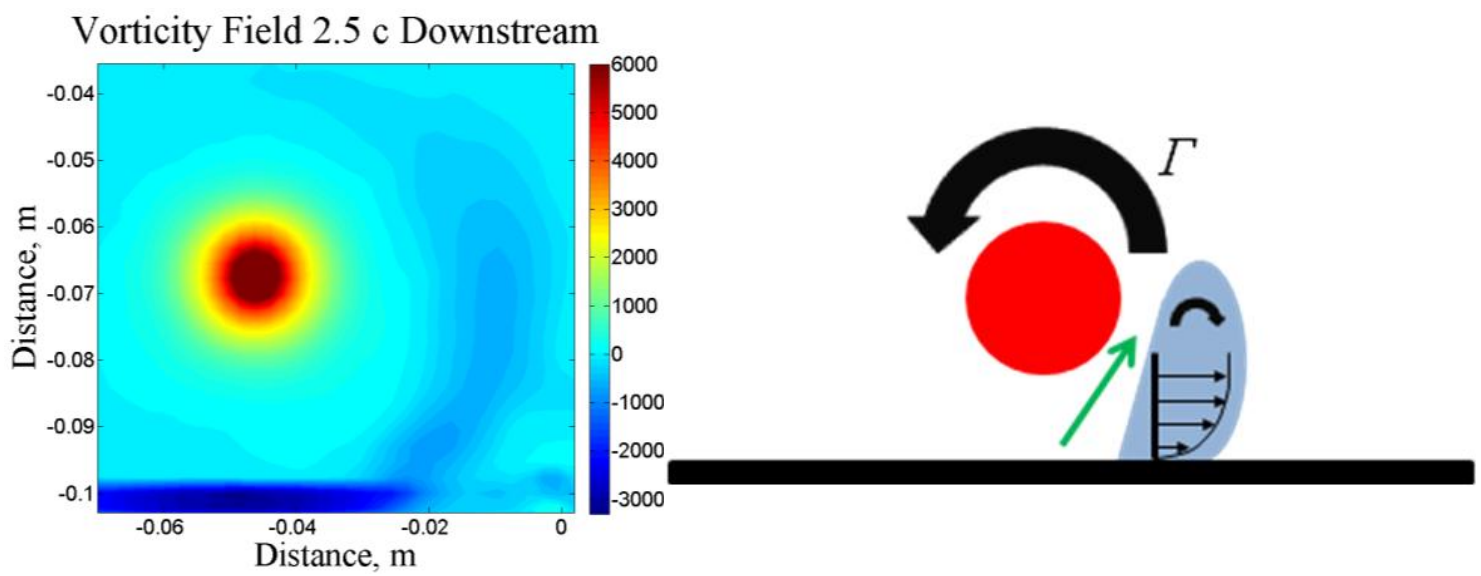

a)

b)

Figure 3.6. (a) Contour plot of vorticity $\left(s^{-1}\right)$ for a gap size of $30 \mathrm{~mm}$ and at a location $2.5 c$ downstream. The opposite signed vorticity from the boundary layer has been convected away from the wall by the vortex. $(b)$ schematic diagram explaining the origins of the rebound effect and of the stream-wise boundary layer. The whole system is convected in the direction of the green arrow.

Another factor which contributes to the increase in $b$ in the $\mathrm{C} 1$ configuration downstream is simply the increase in size of the streamwise boundary layer. A turbulent boundary layer was initiated at the leading edge of the splitter plate, hence the following correlation may be used to model the growth of the turbulent boundary layer

$$
\delta=\frac{0.37 z}{\left(\frac{U_{\infty} z}{v}\right)^{\frac{1}{5}}}
$$

A non-dimensional version of Eq. (A.1) is given by:

$$
t_{b}^{*}=t_{b} \frac{U_{\infty}}{c}
$$

In order to analytically relate the change in $t_{b}{ }^{*}$ due to the distance travelled downstream, it is assumed that the change in characteristic time is solely caused by a change in $b$, and this change in $b$ is caused by the boundary layer growth in the streamwise direction. Combing Eq. (A.1), Eq. (3.2), and Eq. (3.3), it can be shown that $\Delta t_{b}^{*} \sim \Delta z^{*}$, where $z^{*}=z / c$. Figure 3.5 shows that the growth of the characteristic time seems to be linear with downstream distance. This trend is the same as the growth predicted by the simplified boundary layer model. Most likely, the increase in characteristic time is a combination of the boundary layer effect, the rebound effect, and the decrease in circulation as one travels downstream. This implies that if the Crow instability is not present immediately in the flow, it may not develop at any downstream locations (depending on the growth rate), due to the continual increase in the characteristic time.

\section{Numerical Simulations}

Numerical simulations were used to investigate both the two-vortex and one-vortex configurations further. In all of the numerical simulations, a high-order conservative finite difference scheme for the Navier-Stokes equations was used. ${ }^{12}$ From previous studies, it had been determined that using a $4^{\text {th }}$ order accurate scheme for both the convective and viscous terms was required to preserve the physics of the flow. 


\section{A. Two Vortex Simulations}

To study the interactions of two counter-rotating vortices, the numerical simulations are initialized with two counter-rotating Lamb-Oseen vortices. In addition, a velocity, equal and opposite to the speed of the two-vortex system, is superimposed to ensure that the vortices remain stationary during the simulations. Figure 4.1 shows a comparison between an experimentally measured velocity field and the initial velocity field for a numerical simulation. The Lamb-Oseen vortex shape was found to reproduce the experimental vortices well.

The first 3D simulation that was performed consisted of two straight counter-rotating Lamb-Oseen vortices in a periodic domain,

with $L x=L y \sim 20 \mathrm{~b}$. The vortex centers remain constant in the $x$-y plane, and extend in the z-direction. In order to visualize the vortices in a 3D domain, iso-surfaces of $\mathrm{Q}^{13}$ were created, and were colored by the value of vorticity in the $\mathrm{z}$ direction. Figure 4.2 shows the vortices at $t=0.1 \mathrm{~s}$. In the wind tunnel, the time taken by the mean flow to travel from the trailing edge of the airfoils to the final plane of measurement is approximately $0.02 \mathrm{~s}$. The vortices were found to remain stationary for the entire run time, which was much larger than the expected time needed for the Crow instability to develop. This proved that the numerical approach is satisfactory for the present investigation.

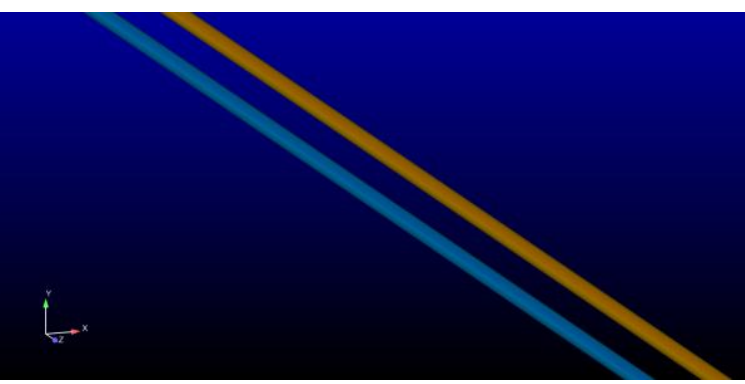

a)

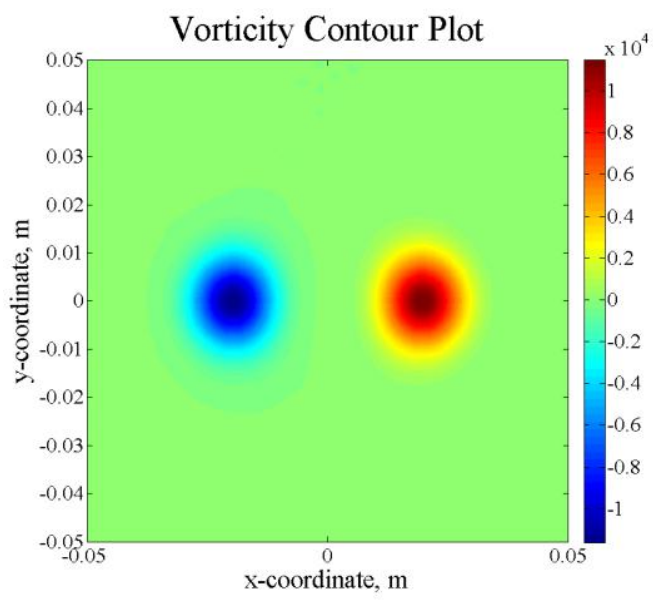

b)

Figure 4.2. 3D simulation of two counter-rotating Lamb-Oseen vortices. a) iso-contours of $Q$ shown, colored by vorticity. at $t=0.1 \mathrm{~s}$. b) vorticity contour plot for one $\mathbf{x}-\mathbf{y}$ plane.

In order to confirm that the Crow instability could be observed numerically, a sinusoidal perturbation corresponding to the theoretical most amplified wavelength was added to the initial conditions. ${ }^{5}$ The center of the vortex was varied in the y direction, with a maximum disturbance of $10 \%$ of the vortex radius. Figure $4.3 \mathrm{c}$ ) shows the evolution of the system at $t=0.015 s\left(3.0 * t_{b}\right.$, corresponding to $3.0 \mathrm{c}$ downstream in the wind tunnel). The initial perturbations have been amplified, and as the simulation continues, these disturbances continue to increase until the vortices eventually connect and break up. It is possible to see a preferred angle of oscillation, and there is a clear plane of symmetry between the vortices. 


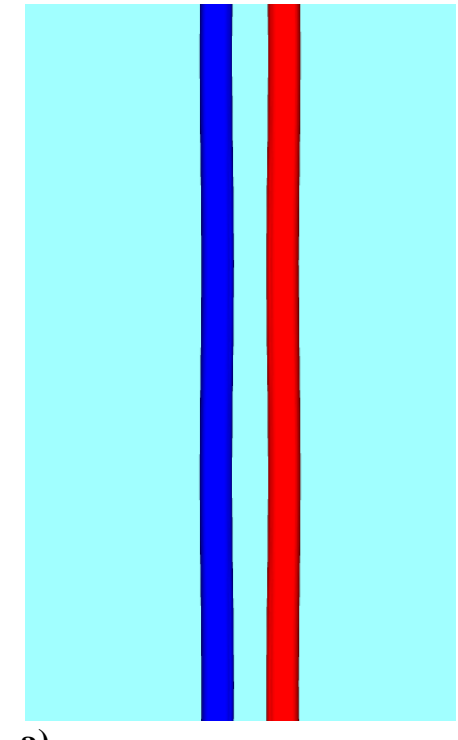

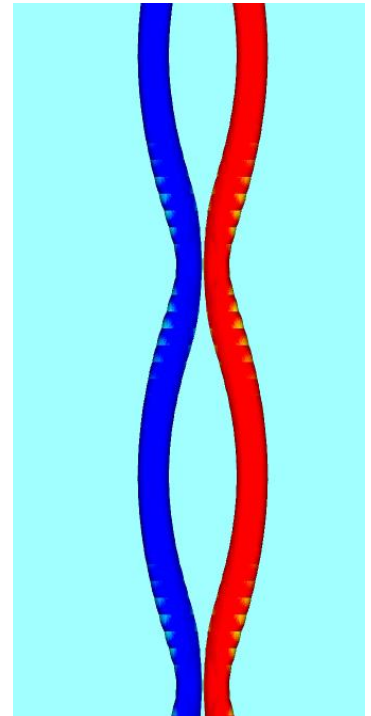

b)

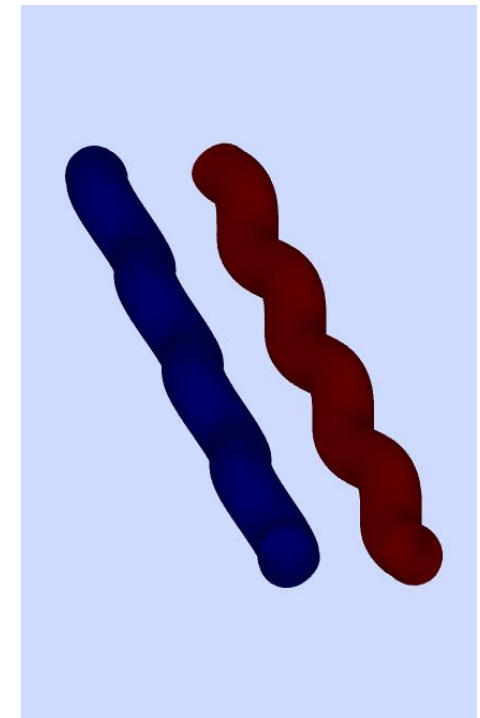

c)

Figure 4.3. 3D simulation of two counter-rotating Lamb-Oseen vortices. Iso-surfaces of $Q$ are shown colored by vorticity in the z-direction. a) top-down view at $0.005 s$. b) top-down view at $0.025 \mathrm{~s}$, showing the growth of the perturbations. c) this snapshot is taken at $t=0.015 \mathrm{~s}$, and a preferred angle of oscillation of approximately $45^{\circ}$ can be seen.

Another way to visualize the evolution of the Crow instability is to perform a similar analysis that was performed earlier with the experimental data. The same center-detection method that was used to analyze the PIV data is applied to each z-plane cut for a numerical simulation. At the beginning of the simulation, the $0^{0}$ angle is consistent with the direction of the initial perturbation imposed. As the system evolves, for the most unstable wavelength, we expect to observe an angle which is close to $45^{\circ}$. Figure 4.4 shows the temporal evolution of the principal angle calculated. After an initial transient period, the average angle is $44^{0} \pm 5^{0}$.

Principal Angle of Oscillation vs Time for the Crow Instability (most unstable wavelength)

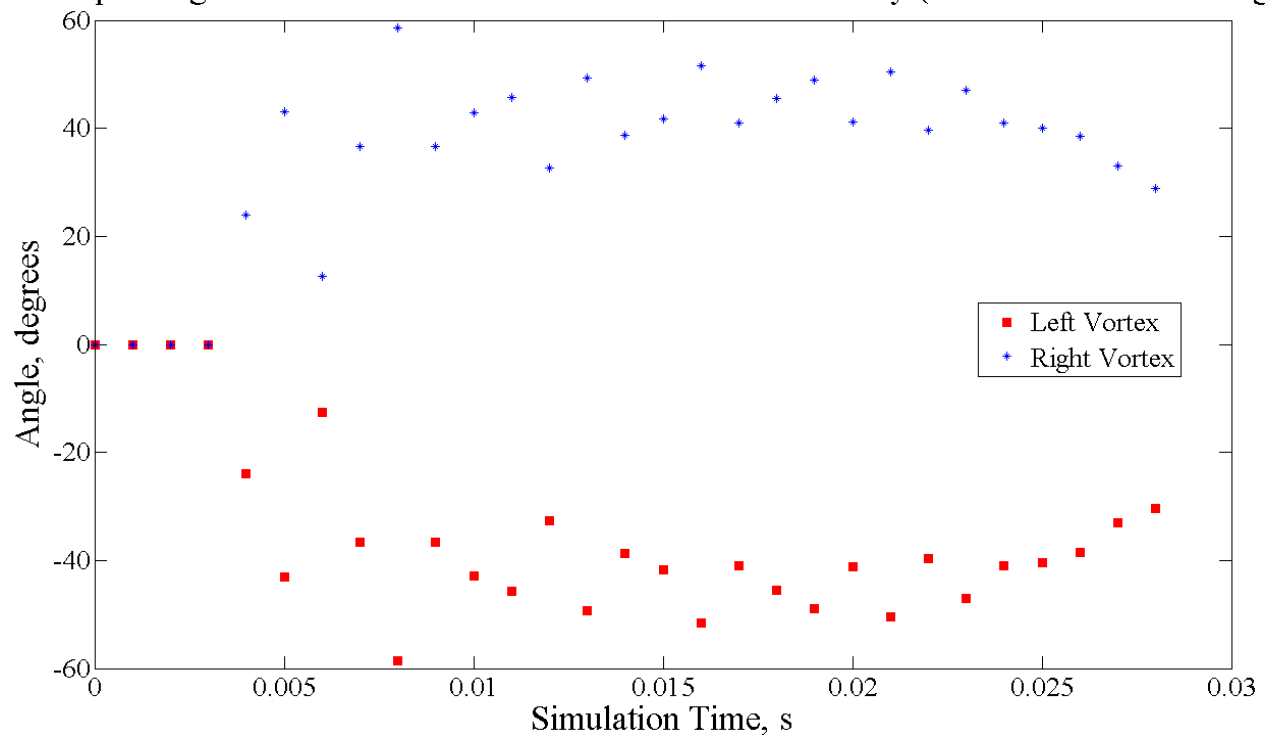

Figure 4.4. Evolution of the principal angle of oscillation. After an initial start-up period, the angle remains reasonably close to $45^{\circ}$ until the vortices begin to break up. 


\section{B. One Vortex and a Wall Configuration}

Another set of simulations were performed with a single Lamb-Oseen vortex, and a no-slip wall at the bottom of the domain. Once again, the domain was chosen to be large enough in comparison to the size of the vortex $(L x \sim 75 a, L y \sim 65 a)$, and large in comparison to the separation distance between the vortex and the wall, which was $4 a$, to ensure that the boundary conditions had no effect on the simulation.

Figure 4.5 shows a cross section of a single vortex taken $0.0125 s$ into the numerical simulation. This figure should be compared with Fig. 3.6 which shows experimental results for $\mathrm{C} 1$ configuration. One of the main differences between the experimental results and the numerical results is that, in the experiments, a large region of opposite signed vorticity is convected away from the wall by the primary vortex. However, in the numerical simulations, smaller secondary vortices are actually rolled up from the wall. This difference is believed to be caused by the initial velocity field imposed in the simulations. There is no boundary layer next to the wall in the initial velocity profile in the numerical simulation. Therefore, the initial velocity field is not continuous at the wall, and this could result in discrete patches of vorticity being convected away from the wall, instead of a smooth region of opposite signed vorticity, as seen in the experiments. It is also possible to see some numerical oscillations originating from the boundary layer in Fig. 4.5, and ways to eliminate these oscillations are currently being investigated.

The presence of a wall fundamentally changes the physics of this vortex system. Even when a perturbation is added to the initial vortex near a wall, the Crow instability does not develop as it did previously with two counterrotating vortices. Instead, the only way the initial vortex can break up is after a second vortex of opposite signed vorticity has fully been rolled-up from the wall, and the two are allowed to interact. However, due to the relatively long amount of time required for this interaction to occur, it would not be of practical use when trying to improve compressor efficiency.

\section{Ongoing Work}

Many other aspects are currently being investigated, mainly focusing on the numerical aspect of the study. A more detailed investigation into the temporal evolution of the principal angle of oscillation of the vortices in the two-vortex configuration is being performed. More specifically, the effect of the initial perturbations (white noise, wavelength, direction, etc) are being investigated. With the one-vortex and a wall simulations, work is being done to determine the cause of the numerical oscillations originating in the boundary layer. Also, the effect of the choice of the initial velocity field is being investigated, in hopes of having a better correlation between the numerical and experimental results.

\section{Conclusions}

A counter-rotating vortex pair $(\mathrm{C} 2)$ and a vortex interacting with a wall $(\mathrm{C} 1)$ have been analyzed. Experimental and numerical measurements were performed to determine if the Crow instability was present in these configurations. With the $\mathrm{C} 2$ configuration is was possible to observe the Crow instability, while the Crow instability was not observed with the $\mathrm{C} 1$ configuration. The increase in characteristic time for the Crow instability as one travels downstream in the $\mathrm{C} 1$ configuration implies that if the Crow instability is not present initially, it may never develop downstream. A simplified model involving the streamwise boundary layer has been proposed to explain the increase in characteristic time with downstream distance for the $\mathrm{C} 1$ configuration. The way in which a single vortex in the vicinity of a wall breaks up is fundamentally different than the break up of two counter-rotating vortices.

13

American Institute of Aeronautics and Astronautics 
Numerical simulations have re-enforced the experimental results, illustrating the fundamental differences in the two systems.

Time-resolved PIV on both configurations is recommended for further work. With time-resolved PIV, it would be possible to examine the spectral component of these systems, which could add much to understanding how these systems evolve and behave. In addition, it would be interesting to consider an airfoil with a higher coefficient of lift, in order to increase the circulation and hopefully reduce the characteristic time enough in the $\mathrm{C} 1$ configuration so that the Crow instability would theoretically have enough time to form. Similarly, additional simulations should be performed varying the vortex distance from the wall and the strength of vortex in order to see if it possible to promote the Crow instability at some point with one vortex and a wall.

\section{Appendix A}

The time scale associated with the Crow instability is given by

$$
t_{b}=\frac{2 \pi b^{2}}{\Gamma}
$$

where $b$ is the distance between the vortex centers, and $\Gamma$ is the magnitude of circulation for each vortex. This time is generally used only as an estimate for the time required for the Crow instability to develop. In practice, several times $t_{b}$ may be necessary before the instability may be detected. This parameter is often taken to be the most important factor to compare different configurations and analyze the dynamics of different vortex systems.

\section{Appendix B}

\begin{tabular}{|c|c|}
\hline \multicolumn{2}{|c|}{ PIV Characteristics } \\
\hline Type of PIV & Stereo (3C) \\
\hline Laser & Nd:YAG Thales PVL 400/TS \\
\hline Cameras & LA Vision Imager Intense \\
\hline Frequency & $4 \mathrm{~Hz}$ \\
\hline Image Size (pixels) & 1376 by 1040 \\
\hline Camera Focal Length & $50 \mathrm{~mm}$ \\
\hline Conversion & 5.0 pixels $/ \mathrm{mm}$ \\
\hline Time between Images & $30 \mu \mathrm{s}$ \\
\hline Laser Sheet Size (usable) & $2 \mathrm{~mm}$ \\
\hline Pixel Shift in Free Stream & 4.5 \\
\hline Vector Spacing & Every 2.4 mm \\
\hline Vortex Diameter/Spacing & $\sim 10$ \\
\hline Images per Trial & 1000 or 2000 \\
\hline Seeding Particles & Magnum Smoke Generator \\
\hline Software Used & LA Vision (DaVis) \\
& FOLKI PIV ${ }^{14}$ \\
& (developed by ONERA) \\
\hline
\end{tabular}

\section{Acknowledgements}

J. Rabinovitch would like to thank the Dual Masters Program between the California Institute of Technology and Ecole Polytechnique, and the Partner University Fund for funding him while performing the experimental component of this research. Also thank you to the members of ONERA DAFE, Meudon, for all of their help with the experiments and the post-treatment of the experimental data. 


\section{References}

${ }^{1}$ Crow, S.C., "Stability theory for a pair of trailing vortices," AIAA Journal, Vol. 8, No. 12, 1970, pp. 2172-2179.

${ }^{2}$ Devenport, W. J., Zsoldos, J. S., and Vogel, C. M., "The structure and development of a counter-rotating wing-tip vortex pair," Journal of Fluid Mechanics, Vol. 332, 1997, pp. 71-104.

${ }^{3}$ Bae, J., Active control of tip clearance flow in axial compressors," Ph.D Dissertation, Dept. of Aeronautics and Astronautics, Massachusetts Institute of Technology, Cambridge, MA, 2001.

${ }^{4}$ Lakshminarayana, B., and Horlock, J., "Tip-clearance flow and losses for an isolated compressor blade," Aeronautical Research Council Reports and Memoranda 3316, 1963.

${ }^{5}$ Brion, V., "Stabilité des paires de tourbillons contra-rotatifs: application au tourbillon de jeu dans les turbomachines," Ph.D. Dissertation, Mécanique Dept., ONERA and Ecole Polytechnique, Meudon, France, 2009.

${ }^{6}$ Tsai, C.-Y., and Widnall, S., "The stability of short waves on a straight vortex filament in a weak externally imposed strain field," Journal of Fluid Mechanics, Vol. 73, 1976, pp. 721-733.

${ }^{7}$ Schlicting, Hermann. Boundary Layer Theory. McGraw-Hill Book Company, New York, 1979.

${ }^{8}$ Graftieaux, L., Michard, M. and Grosjean, N., "Combining PIV, POD and vortex identification algorithms for the study of unsteady turbulent swirling flows," Measurement Science and Technology, Vol. 12, 2001, pp. 1422-1429.

${ }^{9}$ Jeong, J., and hussain, F., "On the identification of a vortex," Journal of Fluid Mechanics, Vol .312, 1996, pp. 67-106.

${ }^{10}$ Devenport, W. J., Rife, M. C., Liapis, S. I., and Follin, G. J., "The structure and development of a wing-tip vortex," Journal of Fluid Mechanics, Vol. 312, 1996, pp. 67-106.

${ }^{11}$ Johnson, R, A., and Wichern, D. W., Applied Multivariate Statistical Analysis, 5th ed., Prentice Hall, New Jersey, 2002, Chap. 8.

${ }^{12}$ Desjardins, O., Blanquart, G., Balarac, B., and Pitsch, H., "High order conservative finite difference for variable density low Mach number turbulent flows," Journal of Computational Physics, Vol. 227, 2008, pp. 7125-7159.

${ }^{13}$ Jeong, J., and F. Hussain. On the indentification of a vortex. J. Fluid Mech. Vol 285 (1995) 69-94.

${ }^{14}$ Le Sant, Y., Leclaire, B., Davoust, S., Champagnat, F., Plyer, A., and Le Besnerais, G., "FOLKI-PIV : une nouvelle approche ultra-rapide pour le calcule champs PIV Stéréo," Congrès Francophone de Techniques Laser, CFTL 2010, Vandoeuvre-lès-Nancy, 2010. 